\title{
Thermal contact conductance and its dependence on load cycling
}

\section{Gopal ${ }^{\mathrm{a}}$, M.J. Whiting ${ }^{\mathrm{a} *}$, J.W. Chew ${ }^{\mathrm{a}}$, S. Mills ${ }^{\mathrm{b}}$}

a. Thermo Fluids Systems University Technology Centre, Faculty of Engineering and Physical sciences, University of Surrey, Guildford, Surrey, GU2 7XH, UK

b. Department of Combustion Engineering, Rolls Royce, Derby, Derbyshire, DE24 8BJ, UK

\author{
* Corresponding author. Tel. +44 1483689611 \\ Email addresses: vj.gopal@gmail.com (V. Gopal), m.whiting@surrey.ac.uk (M.J. Whiting), \\ j.chew@surrey.ac.uk (J.W.Chew), Stephen.Mills@rolls-royce.com (S. Mills)
}

\begin{abstract}
Heat transfer between contacting surfaces is an important factor in the thermal behaviour of engineering components in turbomachinery and various other areas of technology. Thermal contact conductance (TCC) is a parameter that quantifies this heat flow. Any theoretical prediction of TCC should take into account the effects, if any, introduced by repeated loading and unloading. This study aims to add to the limited volume of work available on this topic in the literature. In particular, the focus of this investigation is machined surfaces that typify the mating surfaces in some turbomachinery applications. Experimental work investigating the effect of loading and unloading history for numerous cycles is presented. An instrumented split tube with in line washers, loaded and unloaded under carefully controlled conditions, was used to measure the TCC of washers made of nickel alloy PE16 and 316 stainless steel. The study also examines the load cycle effect on TCC for a variety of interface surface geometries and pressures that are relevant to turbomachinery applications. The results show that load cycling, beyond the first cycle, has a minimal effect on TCC, in disagreement with other studies in the literature. This observation is seen for variety of surface topographies and maximum contact pressures.
\end{abstract}

\section{Keywords}

Thermal Contact Conductance; Load cycling; Thermal conductivity; Turbomachinery. 


\section{Introduction}

When heat flows across the interface between two contacting bodies, a temperature discontinuity occurs at the interface. Thermal contact conductance (TCC) is the parameter that quantifies the conductive heat flow across such contacting interfaces. An understanding, and measurement, of TCC is necessary in a variety of engineering fields such as the automotive, microelectronics, metalworking and gas turbine industries. The importance of this topic is indicated by the publication of major reviews, see for example $[1,2]$.

The effect of surface roughness, material properties and applied load on the first realisation of TCC is well documented in the literature (see, for example, [3-5]). Numerous amount of work has been carried out to study the deformation analysis of the contacting asperities. Among the pioneers of this study are Greenwood and Williamson [6] and Mikic and Rohsenhow [7]. Based on the deformation theories propsed by Greenwood and Williamson and Mikic and Rohsenhow, numerous theoretical studies have attempted to predict the effect of various parameters on the TCC of flat conforming surfaces [7-9]. Greenwood and Williamson [6] describes the deformation of the asperities based on the elastic theory, while Mikic and Rohsenhow [7], Cooper et al. [8], and Yovanovich [9] predict the contact conductance on the assumption that the deformation mode of the contacting asperities are plastic. Little work is available on the TCC of non conforming wavy surfaces [10-12]. A recent study by Gopal et al. [13] demonstrated the importance of understanding the effect of various length scales on TCC of machined mating interfaces in some gas turbine applications. The study proposed a convenient way to model the surface geometries of a machined surface in a simple yet accurate manner. The finite element modelling approach presented by Gopal et al. offers the possibility of estimating the effect of machined geometry on TCC for gas turbine components during loading.

In real applications, a component is exposed to diverse loads and temperature over time. One specific complication is the subjection of an interface to load cycling and the implications for TCC. From the limited literature available, it appears that TCC is 
influenced by operational history. Li et al. [14] conducted a study to enhance the TCC of joints. In their study it was argued that there is an increase in TCC with loading history. Stainless steel was loaded and unloaded for numerous cycles between pressures of 0.845 $\mathrm{MPa}$ to $6.425 \mathrm{MPa}$. Increments in TCC of up to $16 \%$ were observed in the first five load cycles and a further $2 \%$ up to the $30^{\text {th }}$ cycle. A similar study was conducted by Wahid and Madhusudana [15] and a similar conclusion was made. They reported an increment of $10 \%$ in TCC values for the first 25 cycles. Both of these studies argue that the operational effect on TCC is important for at least the first 20 cycles. The load cycle effect was explained in terms of a different deformation mode operating for the contacting asperities during different cycles $[14,15]$. The current study presents data that contradict these earlier findings.

To make valid conclusions about the various factors affecting TCC, it is critically important that the inevitable errors in measurement are calculated realistically. The common experimental set up uses a steady state method with thermocouples embedded in cylindrical bars. The major source of errors associated with this set up is the measurement of the temperature difference between the interfaces in contact. Accuracy of the experiment improves with larger temperature drop across the interface. Another common source of error with this set up is the simplifying assumption that the thermal conductivity is constant across the entire assembly. Large quantities of experimental work available in the literature assume constant thermal conductivity across the entire assembly, for example see $[3-5,14,15]$.

This paper makes use of a rigorous uncertainty analysis and uses a data reduction technique developed originally by Chen [16] to account for the variation of thermal conductivity with temperature. Having addressed these two issues, this paper then investigates the variation of TCC with load cycling for both 316 stainless steel and nickel alloy PE16. Various surface geometries are examined in order to facilitate comparison with previous studies and to extend the work to machined surfaces, which typify those found in some turbo machinery applications. 


\section{Data Reduction Technique for Calculation of TCC}

A detailed description of the experimental procedure, including the methodology for TCC calculation and the surface characterisation of the specimens has been reported by Gopal et al. [13]. A brief description is given below.

A split tube experimental apparatus as shown in Figure 1 was used, where washer specimens are located in between two steel cylinders. Thermocouples are embedded in these steel cylinders for temperature measurement. To generate the axial heat flow, a band heater was fitted to the top steel cylinder and a water cooler was fitted to the bottom steel cylinder. This assembly was insulated using foil wrapped glass wool cladding. The entire rig was placed inside an Instron machine for controlled loading and unloading of the samples. For a detailed explanation of the testing procedure see Woodland [17].

Based on this test set up TCC values are obtained by calculating the heat flux across the entire assembly and dividing it by the temperature drop across the interface as shown in Equation 1. Regression analysis is used to extrapolate the thermocouple measurements to get the steel cylinder interface temperature. This is then used to calculate the temperature drop across the washer interfaces. For a one dimensional steady state heat transfer, without internal heat generation or heat loss, heat transfer can be defined by Equation 2 .

$T C C=\frac{q_{a v g}}{\Delta T}$

Equation 1

$q=k_{t}\left(\frac{d T}{d z}\right)$

Equation 2

Where $k_{t}$ represents the thermal conductivity of the steel cylinder. It is well known that the thermal conductivity of austenitic stainless steel varies with temperature. According to Assael and Gialou [18] the variation of thermal conductivity for stainless steel 304 could be expressed as: 
$k\left(\frac{W}{m K}\right)=5.67+(0.034 T)-\left(1.901 \times 10^{-05} T^{2}\right) \quad$ Equation 3

So integrating Equation 2 the following is obtained:

$$
\int k_{t}(T) d T=q z+C
$$

\section{Equation 4}

Where $q$ is the heat flux, $z$ is the location of the thermocouple along the steel cylinder and $C$ is an integration constant.

As the thermal conductivity varies with temperature, the temperature distribution along the steel cylinder cannot be calculated explicitly. An iterative method based on Chen's [16] numerical technique for processing data was used to develop the solution for heat flux and the temperature distribution along the steel cylinder. Based on Equation 4, $T$ could be represented as:

$T=\frac{q z+C}{\bar{k}_{t}(T)}$

\section{Equation 5}

Where $\bar{k}_{t}(T)=\frac{\int k_{t}(T) d T}{T}$

A function $S$ was defined as the sum of squares of the temperature difference:

$$
S=\sum\left(T_{i e x}-T_{\text {ian }}\right)^{2}
$$

\section{Equation 6}

Where $T_{i e x}$ represents the thermocouple measurement obtained experimentally and $T_{i a n}$ is the analytical temperature distribution along the cylinder. Minimising the function $S$ in terms of $q$ and $C$ and with some algebraic manipulation resulted in an iterative solution for $q$ and $C$ of Equation 4. 


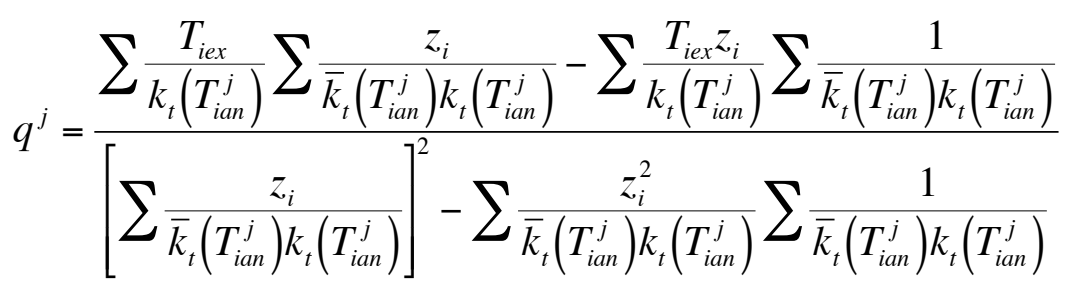

$$
\begin{aligned}
& C^{j}=\frac{\sum \frac{T_{\text {iex }}}{k_{t}\left(T_{\text {ian }}^{j}\right)}-q^{j} \sum \frac{z_{i}}{\bar{k}_{t}\left(T_{\text {ian }}^{j}\right) k_{t}\left(T_{\text {ian }}^{j}\right)}}{\sum \frac{1}{\bar{k}_{t}\left(T_{\text {ian }}^{j}\right) k_{t}\left(T_{\text {ian }}^{j}\right)}}
\end{aligned}
$$

The first estimates for $q$ and $C$ were substituted in Equation 4 to calculate the first estimate of temperature. This was then re substituted to calculate the next realisation of $q$ and $C$. Repeated iteration was carried out until convergence was obtained. Once the converged values of $q$ and $C$ were obtained the steel cylinder interface temperature was calculated.

\subsection{The importance of variation in thermal conductivity for calculating TCC}

The necessity of accounting for the variation in thermal conductivity with temperature can be illustrated. Three test cases were carried out using PE16 nickel washers. These washers were initially lapped to eradicate any flatness variation. The accepted flatness variation for a lapped washer was less than $7 \mu \mathrm{m}$. They were then grit blasted to obtain a specific surface roughness. A Dektak 32 surface profilometer was used to characterise the surface roughness. The surface roughness was determined over an evaluation length of 5 $\mathrm{mm}$. The roughness was characterised by the root mean square (RMS) surface roughness $(\sigma)$ and mean asperity slope $(m)$. The three test cases used different numbers of washers as shown in Table 1.

As can be seen in Table 1 the three sets of washers had very similar surface roughness. Initially TCC was calculated by assuming constant thermal conductivity across the steel cylinder. A thermal conductivity appropriate to the average temperature of the washers 
was chosen for the calculation of heat flux. Solid symbols in Figure 2 represent this initial calculation. The same sets of test data were used to recalculate TCC with the variation of thermal conductivity with temperature taken into consideration. Comparison of these values with the initial ones is shown in Figure 2. For the calculation based on constant thermal conductivity there is a gradual increase in TCC with an increasing number of washers, contrary to what is expected. The temperature variation along the cylinders for test case 3 was about $58.3^{\circ} \mathrm{C}$. Such a temperature difference gives a corresponding variation in thermal conductivity of about $8.7 \%$. While for test case 2 and test case 1 the variation in thermal conductivity is about $8.3 \%$ and $7.9 \%$ respectively. This shows that assuming constant thermal conductivity across the steel cylinder leads to significant errors, which leads to inaccurate TCC values. The effect is larger for higher values of TCC where the temperature drop across the washer pack is smaller and more sensitive to interpolation errors. The results accounting for variable conductivity also show that for the test set up used in this study, TCC measurements were relatively independent of the number of washers in the stack. At the highest pressure, there is a variation in TCC of about $46 \%, 65 \%$ and $85 \%$ for test cases 1,2 and 3 respectively between the two methodologies. This shows that TCC calculation is very susceptible to error if the variation of thermal conductivity with temperature is ignored.

\section{Uncertainty Analysis}

The temperature measurements are taken on the assumption that there is a steady one dimensional temperature field. The overall experimental accuracy depends on the heat loss, accuracy of the temperature measurements and the accuracy of the thermal conductivity chosen to calculate TCC. Heat loss was kept to a minimum by insulating the entire experimental assembly in a foil wrapped glass wool cladding, which ensured the resistance of heat flow to the surroundings was large compared to the interfacial resistance. Any uncertainty in the thermocouple measurements is magnified in the extrapolation to find the interface temperature. The uncertainty of the parameters used for calculating TCC was analysed by conventional error propagation theory. Based on that theory the uncertainty in calculating TCC is shown in Equation 9. 


$$
\delta T C C=\sqrt{\left(\frac{\delta q_{\text {avg }}}{q_{\text {avg }}}\right)^{2}+\left(\frac{\delta T_{W W}}{T_{W W}}\right)^{2}}
$$

\section{Equation 9}

Where $T_{W W}$ is the temperature drop across washer washer interface. The uncertainty of the cylinder interface temperature and the heat flux density is a function of the thermocouple reading and the steel cylinder thermal conductivity. Based on the theory of uncertainty in a function of several variables the uncertainty in the upper interface temperature and heat flux can be expressed as shown in Equation 10.

$$
\begin{aligned}
& \left(\delta T_{h o t}\right)^{2}=\left(\frac{\partial T_{h o t}}{\partial k_{t_{h o t}}} \delta k_{t_{h o t}}\right)^{2}+\left(\delta T_{o}\right)^{2} \sum_{i=1}^{N T}\left(\frac{\partial T_{h o t}}{\partial T_{i}}\right)^{2} \\
& \left(\delta q_{h o t}\right)^{2}=\left(\frac{\partial q_{h o t}}{\partial k_{t_{h o t}}} \delta k_{t_{h o t}}\right)^{2}+\left(\delta T_{o}\right)^{2} \sum_{i=1}^{N T}\left(\frac{\partial q_{h o t}}{\partial T_{i}}\right)^{2}
\end{aligned}
$$

\section{Equation 10}

Where $T_{\text {hot }}$ is the interface temperature of the hot steel cylinder, $k_{t_{h o t}}$ is the thermal conductivity of the hot steel cylinder, $\delta T_{o}$ is the thermocouple accuracy and $N T$ stands for number of thermocouples. Similar expressions hold for the lower (colder) interface temperature. The uncertainty in the measurement of the temperature drop across the washer interface can be expressed as:

$$
\delta \Delta T_{W W}=\sqrt{\left(\delta T_{\text {hot }}\right)^{2}+\left(\delta T_{\text {cold }}\right)^{2}+\left(n \delta T_{W}\right)^{2}}
$$

\section{Equation 11}

Where $T_{W}$ is the temperature drop across the thickness of the washers. The uncertainty of the temperature drop across the washers and the uncertainty in heat flux can be shown to be:

$$
\begin{aligned}
& \delta \Delta T_{W}=\frac{t}{k_{W}} \sqrt{\delta q_{\text {avg }}} \\
& \delta q_{\text {avg }}=\sqrt{\left(\delta q_{\text {cold }}\right)^{2}+\left(\delta q_{\text {hot }}\right)^{2}}
\end{aligned}
$$

\section{Equation 12}


The uncertainty in the overall measurement of TCC is strongly influenced by the uncertainty in the temperature readings and the temperature drop across the interface. Any uncertainty in the thermocouple measurement will be amplified if the temperature drop is lower. Hence, the multiple interface measurement technique used in this study is expected to give better accuracy compared to methods that use only a single interface.

\section{The effect of load cycling on TCC}

This section reconsiders the claim made by some researchers that load cycling has some effect on TCC. This work will also investigate the hysteresis effect that has been seen in previous work. The effect of load cycling on TCC has been attributed to the variation of deformation mode during loading history $[14,15]$. It has already been suggested that such a claim is difficult to substantiate. In order to understand this effect a series of load cycle tests were conducted on two materials with various interface surface geometries. A summary of the tests is shown in Table 2. The two materials used for this study were the nickel alloy PE16 and 316 stainless steel.

\subsection{Test specimens}

The test specimens of PE16 and SS316 were initially turned to an outer diameter of 20 $\mathrm{mm}$ and an inner diameter of $4 \mathrm{~mm}$. These washers were then lapped to a thickness of 2 $\mathrm{mm}$ to obtain similar tolerance as the previous test case. One group of washers was then fly cut on only one side while another group was fly cut on both sides at about 250

$\mu \mathrm{mrev}^{-1}$. This surface geometry was chosen as it has been shown to be similar to some actual mating surfaces in turbo machinery applications, see [13]. The third group of lapped washers were grit blasted to get a rougher conforming surface. The various studies conducted on different interface geometries were to examine whether surface topography has an influence on the change of TCC with load cycling as reported in two previous studies $[14,15]$. 
All sets of washers were characterised using a Veeco Dektak surface profilometer. For unconforming interface geometries, 2D line profiles were obtained in both the machining direction and at $90^{\circ}$ to it. While in the case of conforming surfaces, measurements where obtained in radial and circumferential orientation around the washer. For more detailed information on characterisation of surfaces refer to Gopal et al. [13]. A 3D image of grit blasted, lapped and fly cut surfaces are shown in Figure 3. The three selected surface morphologies represent a wide range of surface topographies. A set of 7 washers stacked between the steel cylinders was used to determine the values of TCC.

\subsubsection{Experimental Procedure}

The test specimens were initially loaded to a maximum nominal pressure of $166 \mathrm{MPa}$ in 4 steps, followed by unloading in the reverse order to a minimum pressure of $33 \mathrm{MPa}$ again in 4 steps. Measurements taken at every step were carried out only after the entire assembly reached thermal steady state. Thermocouple readings were generally recorded for the $10^{\text {th }}, 50^{\text {th }}$, and the $500^{\text {th }}$ cycle. For some cases only the first four cycles were noted. The results for PE16 and SS316 are shown in Figure 4 and 5 respectively.

Figure 4 and 5 both show a clear hysteresis effect for all the test cases irrespective of the interface surface topography except for the lapped lapped contact for PE16 test case. The error bars for this test case during loading and unloading overlap at higher loads, but the trend is very similar to every other test cases. Thus, it is reasonable to conclude that there is a hysteresis effect as expected on the basis of numerous previous studies. The highest variation in TCC between loading and unloading is observed at the lowest pressures, as expected. This is because the highest variation in ratio of the real contact area to the apparent contact area between loading and unloading is expected at the lowest pressure. The percentage difference in TCC values for 316 stainless steel at the lowest pressure for the lapped/lapped contact was about $28 \%$. While for fly cut/lapped, fly cut parallel and fly cut perpendicular contacts the variation was about $64 \%, 49 \%$ and $44 \%$ respectively. For PE16 samples, the variation in TCC at the lowest pressure for the smooth conforming surface contact was $28 \%$. While for the non conforming contacts, fly cut/lapped, fly cut parallel and fly cut perpendicular, the variation was $71 \%, 85 \%$ and $78 \%$ respectively. The 
reason for the difference between smooth conforming contacts and the non conforming contacts could be explained based on the ratio of real contact area to apparent contact area. For smooth conforming surfaces there are more asperities in contact compared to non conforming surfaces, hence the load is distributed over a larger surface area for a smooth conforming surface. As a result the overall deformation of the contacting asperities of the smooth conforming surfaces during initial loading is lower compared to the non conforming contacts. This results in a higher variation of contact area at the same load, during loading and unloading, for a non conforming contact surface compared to the smooth conforming contacts.

Another important observation that can be made is that, after the first unloading, the TCC for any further cycle is similar to the unloading values, within the experimental errors. This shows that there is no measurable increase in TCC after the first unloading in disagreement with two previous studies [14,15]. Any increase in TCC observed by increasing the load cycle is within the errors of the experiment. It is postulated that the observation of load cycle effects made in earlier studies [14,15], may also be within the experimental errors. This is considered further later on.

When two surfaces are in contact, only certain discrete asperities from each surface will contact the other surface. As the load is increased the initially contacting asperities will deform and so the mean distance between the two surfaces decreases. This will introduce newly contacting asperities and as a result the contacting area will increase. Some of the asperities deform only elastically whilst some of them will deform plastically after some initial elastic deformation. This process is on going as the pressure is increased. Once the maximum load is reached and the interface is unloaded, the asperities that deformed plastically do not regain their original shape. This increases the actual contact area at lower loads during unloading compared to the contact area at the same loads on loading. As a result the TCC is higher during unloading compared to loading at the same contact loads, leading to hysteresis effects. Assuming that the surfaces are not separated and they are loaded again, only the asperities that were in contact at the end of the first unloading will be in contact for the following new cycle. As the asperities that were in contact after the first unloading have already plastically deformed, for the same contact loads they will 
not deform any further than it already has after the first unloading. This is expected to hold true as long as the contact loads at any given loading history do not exceed the maximum load of the first cycle. This argument suggests that thermal contact conductance for a given load should remain constant after the first loading unloading cycle, as observed from the results presented.

In order to understand the effect of maximum load applied in the first cycle on operational history, another test was conducted on PE16 nickel alloys and SS316 at lower pressures. The test carried out was similar to the study conducted in references $[14,15]$. The samples were grit blasted to get a rough surface finish. A summary of the surface parameters and the material properties of the test specimen for the present study and those used in references $[14,15]$ are shown in Table 3 . The specimens were loaded from 0.86 $\mathrm{MPa}$ to $6.4 \mathrm{MPa}$ in 3 steps, followed by unloading back to $0.86 \mathrm{MPa}$ in 3 steps. This was carried out for a maximum of twenty cycles. The result is shown in Figure 6. The results show a clear hysteresis effect while unloading only after the first cycle. From Figure 6 it is noted that no measurable hysteresis effect is seen under the second unloading, giving further support to the hypothesis explained previously.

Figure 7 shows the variation in non dimensionalised conductance with load cycle at the highest pressures. TCC has been non dimensionalised based on Equation 13.

$$
\begin{array}{ll}
C_{C}=\frac{T C C \sigma_{q}}{k m_{a}} \quad \text { Equation } 13 \\
\end{array}
$$

Where $\sigma_{q}$ is the effective RMS roughness, $m_{a}$ is the effective mean asperity slope and $k$ is the thermal conductivity of the material. It can be noted that for the present study at the highest pressure there is an increase of $10 \%$ in TCC value from the first loading to the second loading, which is within the errors of the experiment. The value remains constant from the second loading onwards. This shows no significant change in TCC at higher pressures after the first loading. The results are compared with the study conducted by Li et al. [14] and Wahid and Madhusudana [15]. Li et al. came to a conclusion that the 
increase in TCC was about $16 \%$ within the first five cycles and it increases up to $17 \%$ by the $20^{\text {th }}$ cycle. This is a significant increase in TCC at least within the first 5 cycles, but it is not clear what the increase in TCC was from the second cycle onwards. It could be argued that the increment in TCC should have taken place during the first unloading process and any further increment should be within the experimental errors. Similar observation can be made for the result presented by Wahid and Madhusudana [15]. The increase in TCC after the second cycle was about $7.4 \%$ and it increases up to $11 \%$ by the $20^{\text {th }}$ cycle. Uncertainty of both the studies is not clear but there is enough evidence to suggest that there is no increment in TCC after the first unloading. Any further increment after the first unloading could lie within the experimental errors. This appears likely as the experimental set up in both the studies $[14,15]$ used single interface tests, which may have higher errors than the multiple interface technique.

\section{Conclusion}

An improved method of calculating TCC values has been explored in this paper. This methodology takes into consideration the varying thermal conductivity across the test assembly for calculating TCC. The results shown in section 2.1 demonstrates the importance of this methodology. This study has also looked at the effect of operational history on TCC measurement. A clear hysteresis effect was seen only after the first cycle for the two different materials tested. The hysteresis effect seen was related to the different deformation mode during the first loading and unloading. It was also noted that the effect of hysteresis dependent on contacting surfaces. The effect was magnified for non conforming surfaces compared to smooth conforming surfaces. Contrary to previous studies $[14,15]$, no further increment in TCC was noted above the first loading/unloading cycle. The operational effect observed at higher cycles in the literature study has been related to the uncertainty of the results. It was also shown that the load cycle was independent of the material, interface surface topography and the maximum contact pressure. 


\section{Nomenclature}

$\begin{array}{ll}C & \text { Integration constant } \\ C_{c} & \text { Non-dimensionalised conductance } \\ k & \text { Thermal conductivity } \\ m & \text { Mean asperity slope } \\ m_{a} & \text { Effective mean asperity slope } \\ n & \text { Number of washers } \\ N T & \text { Number of thermocouple } \\ q & \text { Axial heat flux } \\ T & \text { Temperature } \\ \text { TCC } & \text { Thermal Contact Conductance } \\ t & \text { Thickness of washers } \\ z & \text { Location co-ordinate along steel cylinder axis } \\ \Delta T & \text { Temperature drop } \\ \delta k & \text { Uncertainty of thermal conductivity } \\ \delta q & \text { Uncertainty of heat flux } \\ \delta T & \text { Temperature uncertainty } \\ \delta T_{o} & \text { Uncertainty of thermocouple reading } \\ \delta T C C & \text { Uncertainty of TCC value } \\ \sigma & \text { RMS surface roughness } \\ \sigma_{q} & \text { Effective RMS surface roughness } \\ & \end{array}$

\section{Subscripts}

an analytical

avg Average value of hot and cold cylinder

cold Lower steel cylinder

ex experimental

hot Upper steel cylinder

$i \quad$ The $\mathrm{i}^{\text {th }}$ thermocouple location 


$\begin{array}{ll}T W & \text { Tube washer interface } \\ t & \text { Steel cylinder } \\ W & \text { Washer } \\ W W & \text { Washer-washer interface }\end{array}$

\section{Superscripts}

$j \quad$ The $\mathrm{j}^{\text {th }}$ iteration 


\section{Reference}

[1] C. Madhusudana, L.S. Fletcher, Contact heat trasnfer - the last decade, AIAA Journal 24 (1986) 510-523.

[2] M. Yovanovich, Four decades of research on thermal contact, gap and joint resistance in microelectronics, IEEE Transactions on components and packaging technologies 28 (2) (2005).

[3] S. Woodland, A.D. Crocombe, J.W. Chew, S.J. Mills, A new method for measuring thermal contact conductance - experimental technique and results, Journal of engineering for gas turbines and power 133 (2011).

[4] S. Kumar, K. Ramamurthi, Thermal contact conductance of pressed contacts at low temperature, Cryogenics 44 (2003) 727-734.

[5] R. Xu, L. Xu, An experimental invesitigation of thermal contact conductance of stainless steel at low temperatures, Cryogenics 45 (2005) 694-704.

[6] J. Greenwood, J. Williamson, Contact of nominally flat surfaces, 295 (1442) (1966) $300-319$.

[7] B. Mikic, W. Rohsenhow, Thermal contact conductance, Technical report for NASA: NGR 22-0090065, MIT, 1966.

[8] M. Cooper, B. Mikic, M. Yovanovich, Thermal contact conductance, International Journal of heat and mass transfer, 12 (3) (1969) 279-300.

[9] M. Yovanovich, Thermal contact correlations, AIAA 83 (1982) 83-95.

[10] M.A. Lambert, L.S. Fletcher, Thermal contact conductance of spherical rough metals, Journal of heat transfer 119 (4) (1997) 684-690.

[11] A.M. Clausing, B.T. Chao, Thermal contact resistance in a vacuum environment, Journal of heat transfer 87 (2) (1965) 243-251.

[12] M. Yovanovich, Thermal contact conductance of turned surfaces, AIAA (1971) 7180.

[13] V. Gopal, M. Whiting, J.W. Chew, S.J. Mills, Finite element analysis as a tool for calculating the thermal contact conductance for a machined surface, Proceedings of ASME summer heat transfer conference, Puerto Rico, 2012.

[14] Y. Li, C. Madhusudana, E. Leonardi, On the enhancement of the thermal contact conductance: Effect of loading history, Journal of heat transfer 122 (2000) 46-49. 
[15] S. Wahid, C. Madhusudana, Thermal contact conductance: effect of overloading and load cycling, International Journal of heat and mass transfer 46 (2003) 4139-4143.

[16] X. Chen, Precision cold forming modelling, interfacial thermal parameter investigation and tool design optimisation, $\mathrm{PhD}$ thesis, University of Strathclyde, Glasgow, 2002.

[17] S. Woodland, Measurement and Modelling of Thermal Contact Conductance, PhD thesis, University of Surrey, Surrey, 2009.

[18] M. Assael, K. Gialou, Measurement of thermal conductivity of stainless steel AISI 304L up to 550k, International Journal of Thermophysics 24 (2003). 
Figure list

Figure 1 Schematic of experimental set up.

Figure 2 TCC comparison for different calculation methodology

Figure 3 3D image of a) grit blasted surface (left); b) lapped surface (middle); c) fly cut surface (right).

Figure 4 TCC of PE16 for contact between: a) lapped-lapped (top left); b) fly cutlapped (top right); c) fly cut-fly cut parallel orientation (bottom left); d) fly cut-fly cut perpendicular orientation (bottom right).

Figure 5 TCC of SS316 for contact between: a) lapped-lapped (top left); b) fly cutlapped (top right); c) fly cut-fly cut parallel orientation (bottom left); d) fly cut-fly cut perpendicular orientation (bottom right).

Figure 6 TCC at low loads for grit blasted interface contact: a) PE16 (left); b) SS316 (right).

Figure 7 Load cycle effect on TCC for SS316 grit blasted surface contact at an operational pressure of $0.86 \mathrm{MPa}$ and 6.4MPa. 


\section{List of Tables}

Table 1 Details of the three test cases.

\begin{tabular}{|c|c|c|c|}
\hline Specimen & PE16 Nickel alloy & $\begin{array}{c}\text { RMS Roughness } \\
(\sigma-\mu m)\end{array}$ & $\begin{array}{c}\text { Mean asperity } \\
\text { slope }(m)\end{array}$ \\
\hline Test case 1 & 4 washers & 0.85 & 0.157 \\
\hline Test case 2 & 7 washers & 0.84 & 0.159 \\
\hline Test case 3 & 10 washers & 0.76 & 0.147 \\
\hline
\end{tabular}

Table 2 Interface surface geometry data of the test specimens

\begin{tabular}{|c|c|c|}
\hline Specimen & PE16 & SS316 \\
\hline 1 & lapped-lapped & lapped-lapped \\
\hline 2 & Fly cut - lapped & Fly cut - lapped \\
\hline 3 & $\begin{array}{c}\text { Fly cut - fly cut (parallel } \\
\text { orientation) }\end{array}$ & Fly cut - fly cut (parallel orientation) \\
\hline 4 & $\begin{array}{c}\text { Fly cut - fly cut (perpendicular } \\
\text { orientation) }\end{array}$ & $\begin{array}{c}\text { Fly cut - fly cut (perpendicular } \\
\text { orientation) }\end{array}$ \\
\hline 5 & Grit blast - Grit blast & Grit blast - Grit blast \\
\hline
\end{tabular}

Table 3 Roughness parameters and material properties of the test specimen.

\begin{tabular}{|c|c|c|c|c|}
\hline & Material & $\begin{array}{c}\text { Effective Surface } \\
\text { roughness- } \\
\sigma_{q}(\mu m)\end{array}$ & $\begin{array}{c}\text { Effective } \\
\text { Mean asperity } \\
\text { slope } \\
m_{a}(\mathrm{rad})\end{array}$ & $\begin{array}{c}\text { Thermal } \\
\text { conductivity } \\
\left(\mathrm{Wm}^{-1} \mathrm{~K}^{-1}\right)\end{array}$ \\
\hline Present work & SS316-SS316 & 3.17 & 0.195 & 14.95 \\
\cline { 2 - 5 } & PE16-PE16 & 3.11 & 0.185 & 12.92 \\
\hline Wahid and & SS304-SS304 & 7.6 & 0.36 & 15 \\
\hline Madhusudana [15] & SS54-SS137 & 2.9 & 0.47 & 12.72 \\
\hline Li et al. [14] & & & & \\
\hline
\end{tabular}


Figure 1

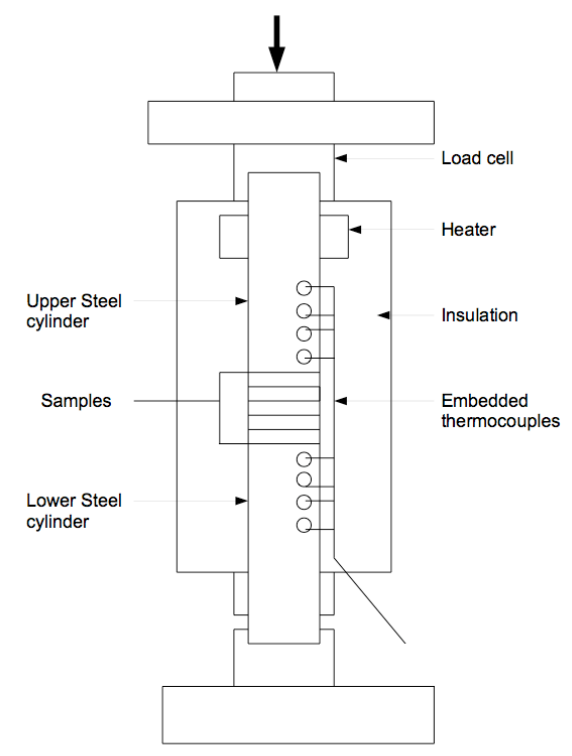

Figure 2

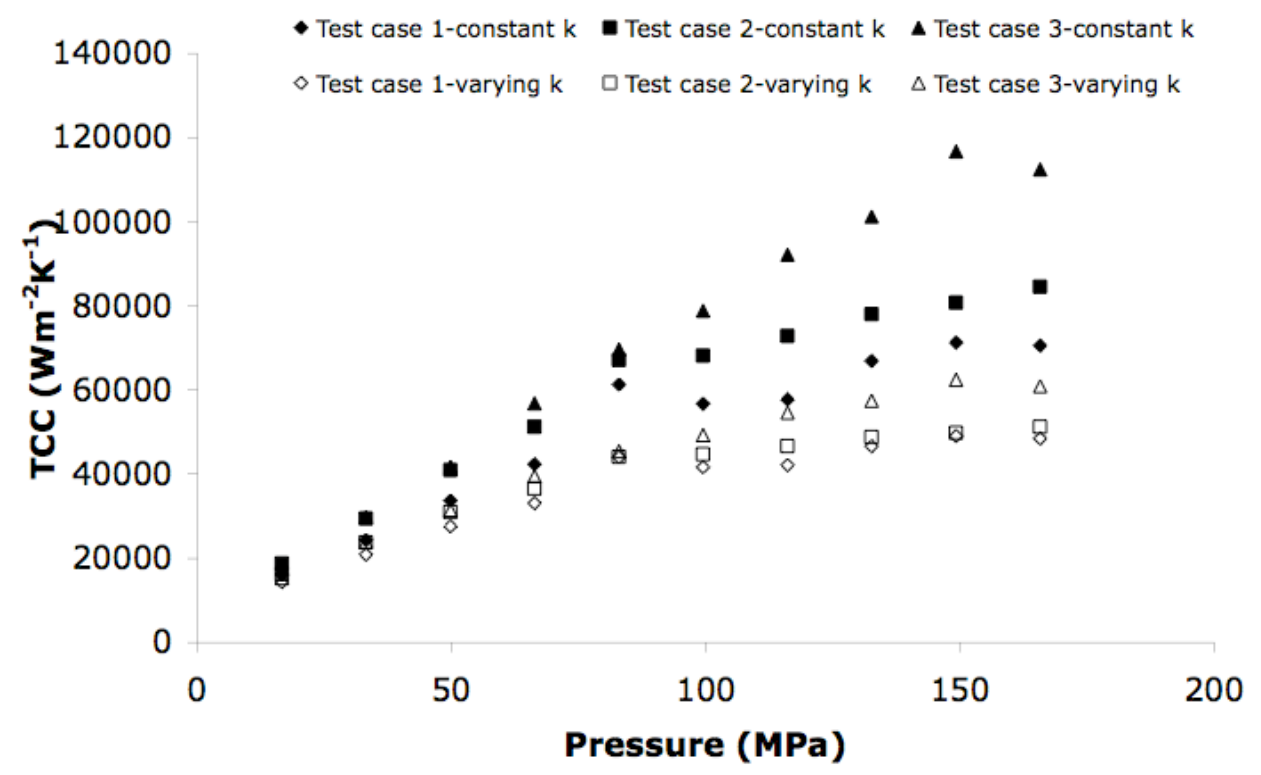


Figure 3

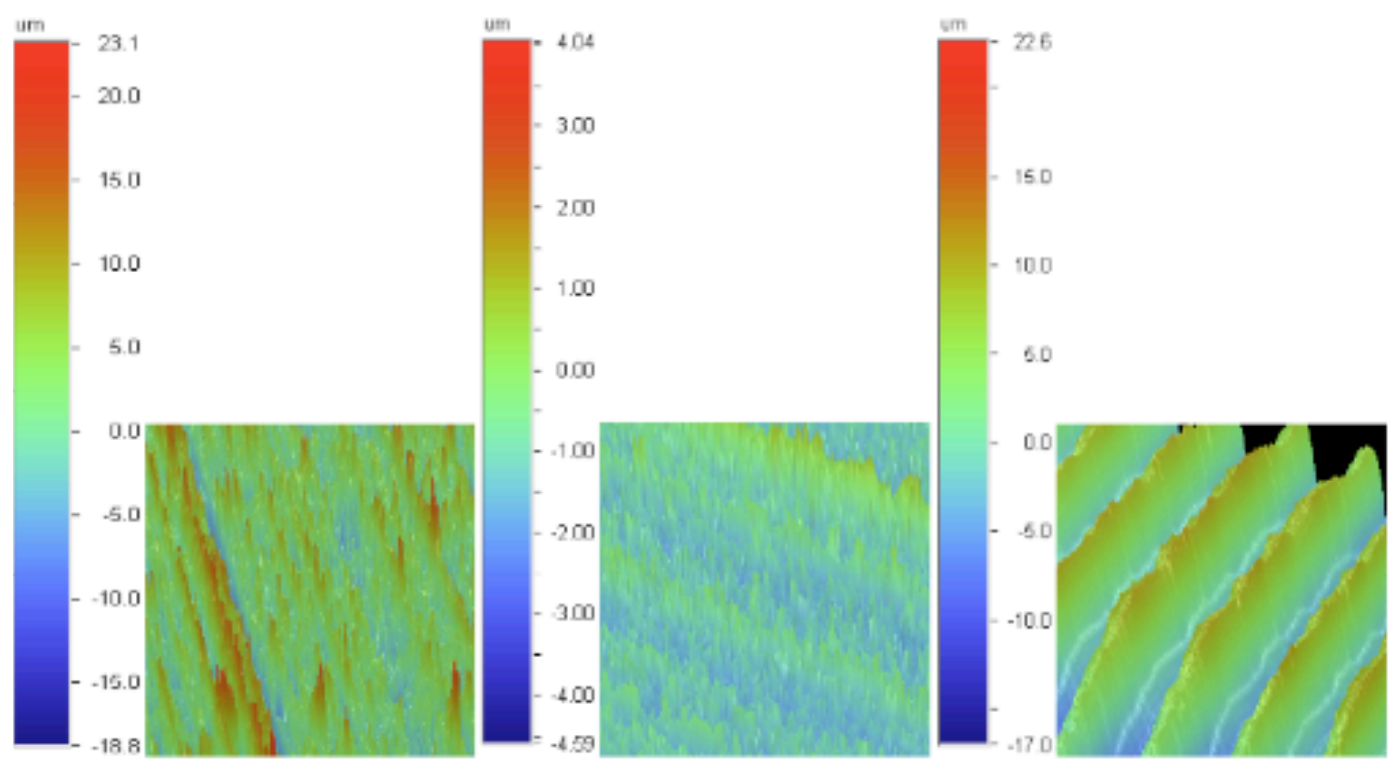

Figure 4
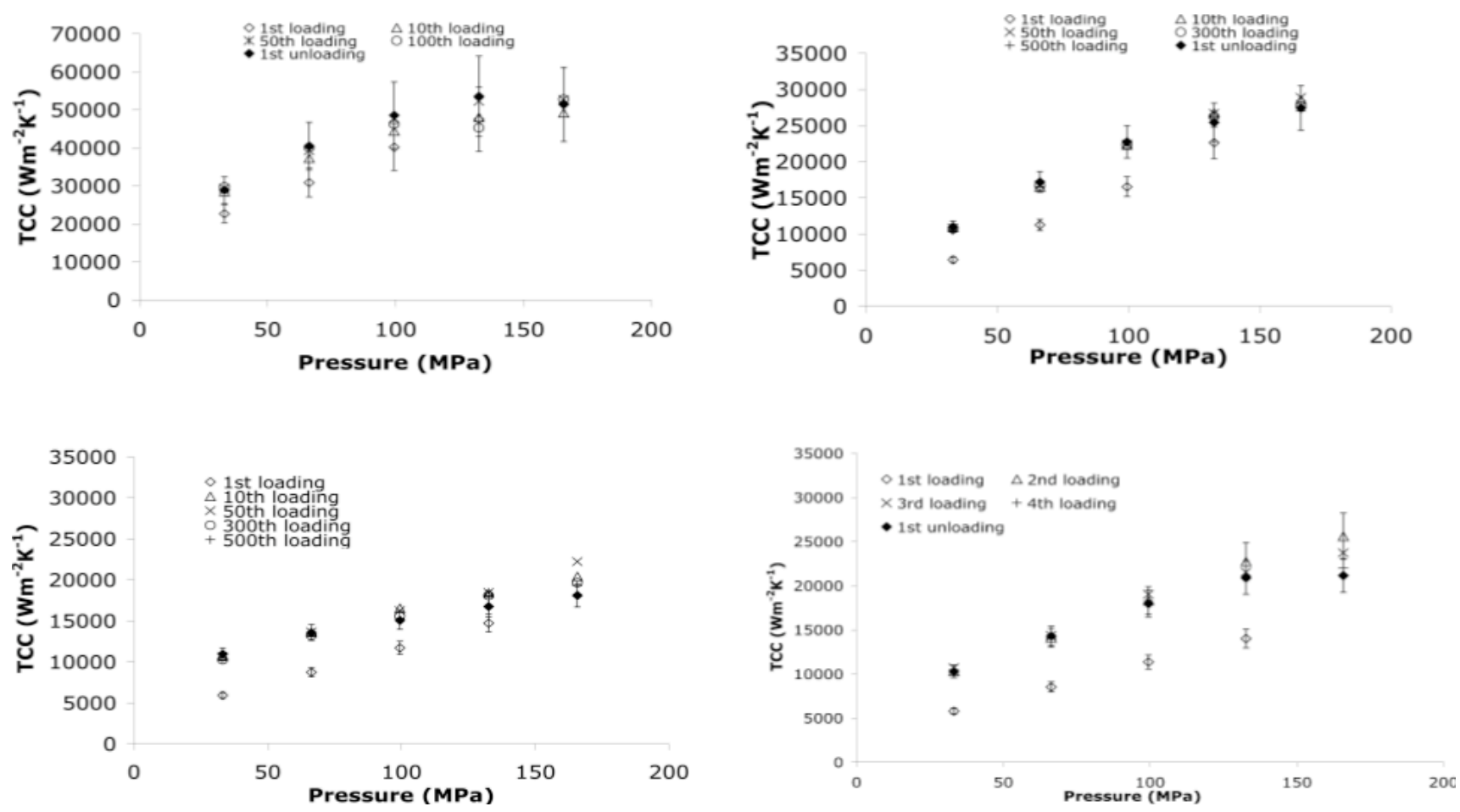
Figure 5
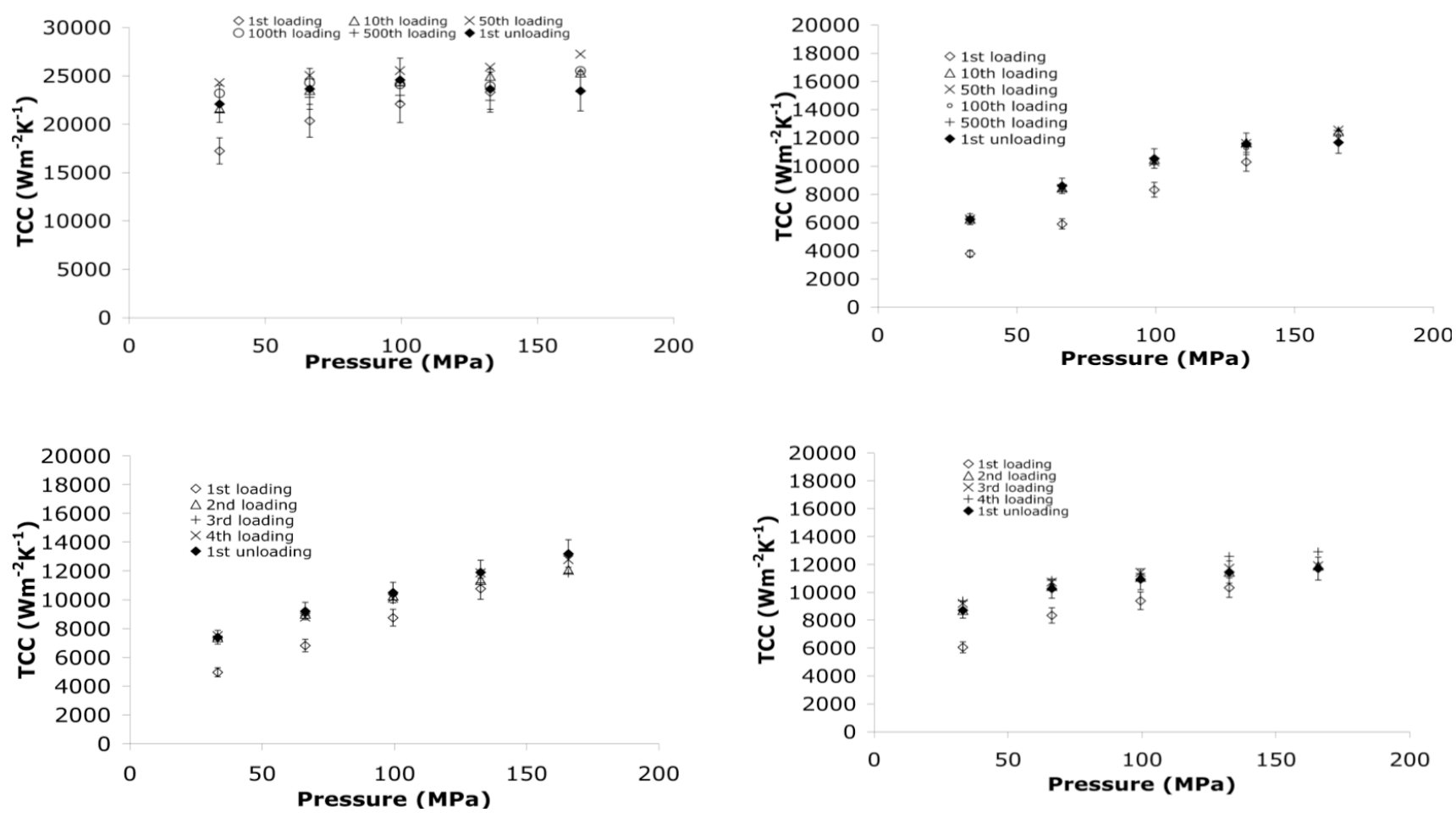

Figure 6
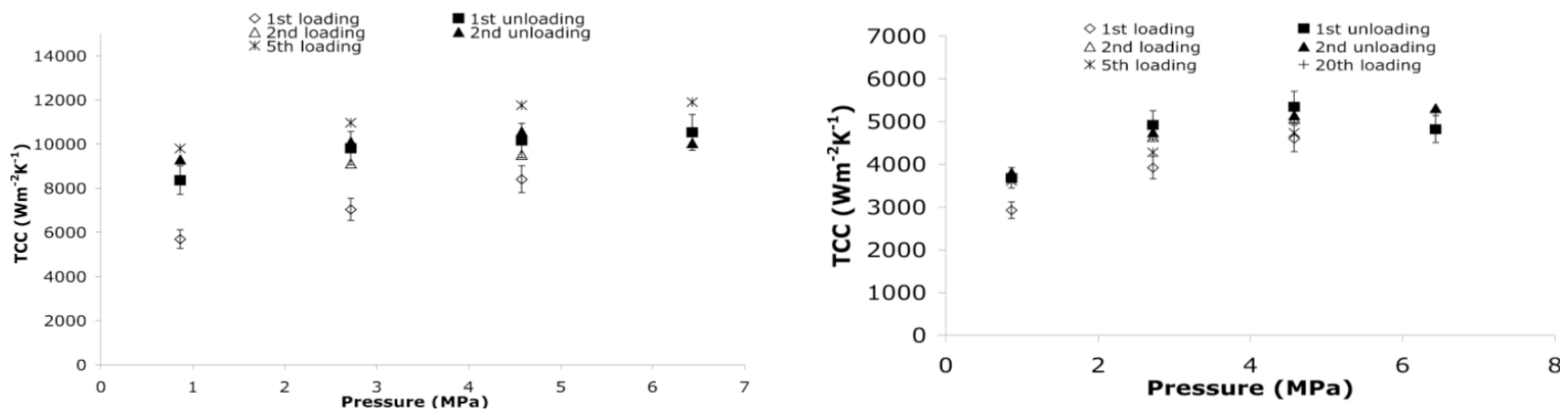
Figure 7

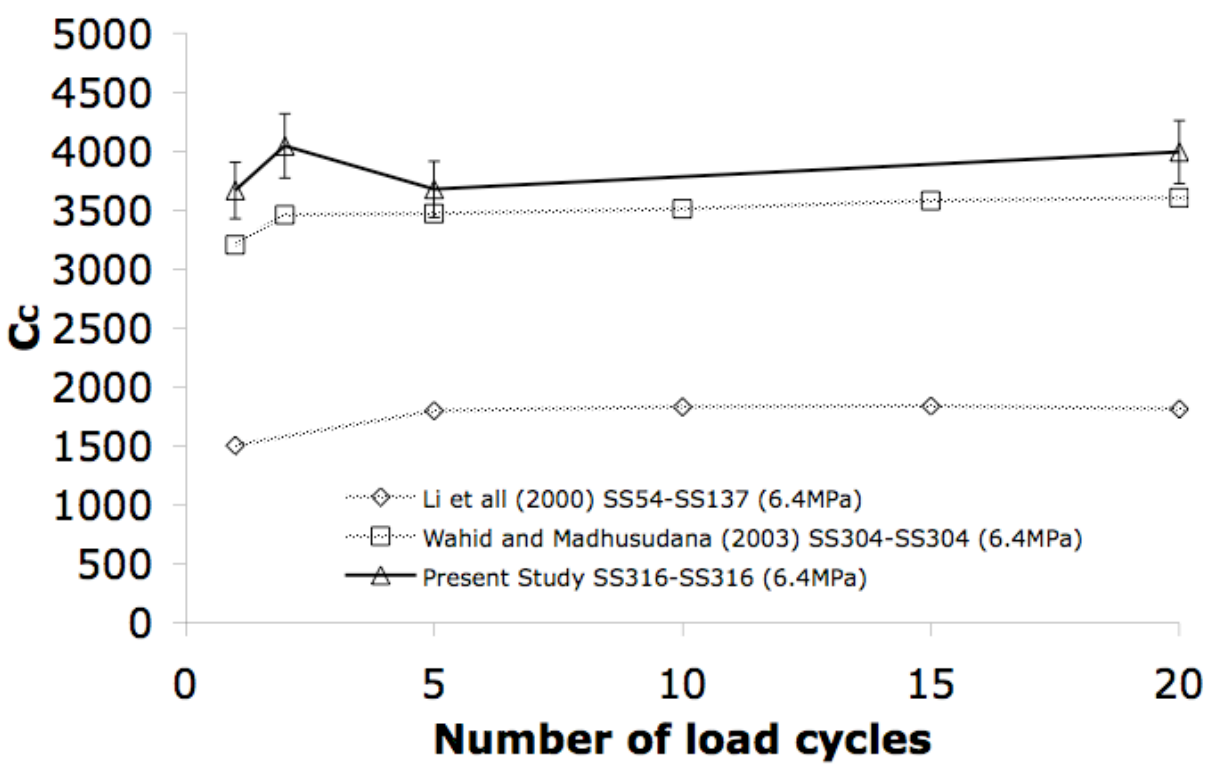

